\title{
Akutes Leberversagen
}

\author{
K. H. W. BöKeR · M. P. MANnS
}

\author{
46.1 Ätiologie 473 \\ 46.1.1 Akute Virushepatitiden 474 \\ 46.1.2 Medikamente 475 \\ 46.1.3 Sonstige Ursachen 476 \\ 46.2 Prognose 476 \\ 46.3 Klinische Probleme und Therapie 477 \\ 46.3.1 Enzephalopathie 477 \\ 46.3.2 Hirnödem 478 \\ 46.3.3 Kardiovaskuläres System und Hämodynamik 479 \\ 46.3.4 Nierenversagen 480 \\ 46.3.5 Pulmonale Komplikationen 480 \\ 46.3.6 Gerinnungsstörung 481 \\ 46.3.7 Metabolische Störungen 481 \\ 46.3.8 Ernährung bei Leberversagen 482 \\ 46.3.9 Sepsis 482 \\ 46.4 Spezifische Therapien 483 \\ 46.5 Prognostische Faktoren 483 \\ 46.6 Leberersatzverfahren 483 \\ 46.6.1 Filtrationsverfahren 484 \\ 46.6.2 Bioreaktoren 484 \\ 46.7 Lebertransplantation 484 \\ 46.7.1 APOLT 485
}

A Is akutes Leberversagen bezeichnet man den Ausfall der Leberfunktion bei Patienten die vorher keine chronische Leberkrankheit hatten. Diese Definition trennt das akute Leberversagen von Endstadien chronischer Leberkrankheiten, bei denen es ebenfalls zum Leberausfall kommen kann. Stellt sich ein solcher terminaler Leberausfall bei chronischer Leberkrankheit als rasch progredientes Ereignis dar, spricht man gelegentlich von einem "akut auf chronischen" Leberversagen. Strenggenommen handelt es sich dabei aber nicht um ein akutes Leberversagen im eigentlichen Sinne.

Im angelsächsischen Sprachraum spricht man von ,acute hepatic failure“, „fulminant hepatic failure" oder auch von „acute liver failure“. Diese Begriffe werden weitestgehend synonym verwendet.

Das akute Leberversagen wird anhand einer typischen Befundkonstellation definiert. Es handelt sich dabei um die Kombination aus Ikterus und Gerinnungsstörung als Zeichen der Leber- insuffizienz und einer Bewußtseinsstörung bei hepatischer Enzephalopathie. Eine so präzise Definition ist von erheblicher klinischer Bedeutung. Die schlechte Spontanprognose des akuten Leberversagens tritt erst in dem Moment ein, in dem die Enzephalopathie zu der schweren Leberfunktionsstörung hinzutritt (O'Grady 1996).

Das akute Leberversagen kann darüber hinaus nach der Dynamik seiner Entwicklung noch weiter unterteilt werden. Man spricht von:

- fulminantem Leberversagen, wenn zwischen dem Ausfall der Leberfunktion und dem Beginn der Enzephalopathie weniger als 7 Tage liegen,

- akutem Leberversagen bei einer Zwischenzeit von 8-28 Tagen und

- subakutem oder protrahiertem Leberversagen, wenn zwischen Ikterus und Enzephalopathie mehr als 4 Wochen vergangen sind.

Diese Untereinteilung ist zur Einschätzung der Prognose hilfreich, hat sich jedoch im praktischen Sprachgebrauch nicht allgemein durchgesetzt (Hoofnagle et al. 1995).

\section{1 \\ Ätiologie}

Die häufigsten Ursachen (siehe Übersicht) eines akuten Leberversagens in Deutschland sind virale Hepatitiden und Medikamententoxizität.

Zu den selteneren Ursachen zählen Knollenblätterpilzvergiftungen, akute Manifestationen des Morbus Wilson und das Budd-Chiari-Syndrom. Man beobachtet hierbei deutliche geographische Unterschiede. So ist in Großbritannien die Paracetamolvergiftung die häufigste Ursache für ein Leberversagen, in den Mittelmeerländern dominiert die Hepatitis B und in Deutschland sehen wir besonders oft idiosynkratische Medikamentenreaktionen (Williams 1996). 


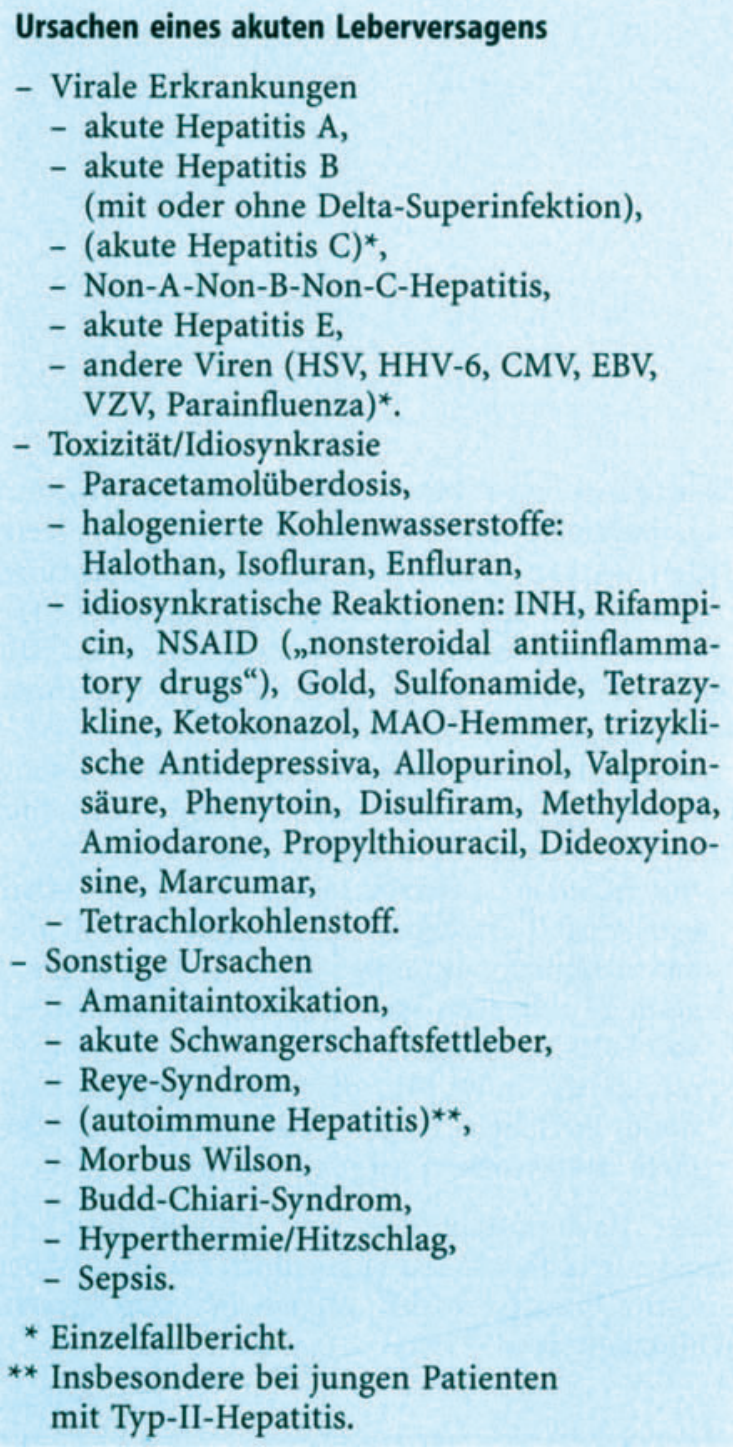

46.1.1

Akute Virushepatitiden (s. Kap. 41)

\section{Hepatitis A}

Bei Hepatitis A wird ein akutes Leberversagen relativ selten gesehen, nämlich bei etwa $0,1-0,4 \%$ der hospitalisierten Patienten (O'Grady 1992).

Da wiederum nur ein kleiner Teil der Patienten mit Hepatitis A überhaupt hospitalisiert wird, dürfte die tatsächliche Häufigkeit noch niedriger liegen. Trotzdem ist diese Krankheit in nordeuropäischen Ländern für bis zu $20 \%$ der Fälle von akutem Leberversagen verantwortlich. Dies dürfte bedingt sein durch den schwereren Verlauf der Erkrankung bei Infektion im Erwachsenenalter.
Pathogenese. Die Pathogenese des akuten Leberversagens bei Hepatitis-A-Virus-Infektion ist nicht vollständig geklärt. Zwar wird das HAV als direkt zytopathologisch angesehen, neuere Befunde deuten aber daraufhin, daß neben der direkt zellschädigenden Wirkung ein wahrscheinlich durch zytotoxische T-Zellen vermittelter Immunprozess für die akute Zerstörung der Leber verantwortlich sein könnte (Vallbracht et al. 1986).

\section{Hepatitis B}

Bei Hepatitis-B-Infektion ist das Risiko, ein akutes Leberversagen $\mathrm{zu}$ entwickeln, höher als bei der Hepatitis A. Etwa $1 \%$ aller hospitalisierten Hepatitis-B-Patienten erleiden diese schwerste Verlaufsform (Hoofnagle et al. 1995). Frauen sind gefährdeter als Männer, besonders wenn gleichzeitig eine Hepatitis-D-Infektion vorliegt. Dabei sind allerdings die meisten Fälle auf eine Delta-Superinfektion bei chronischer Hepatitis B zurückzuführen, im Sinne eines „akut auf chronischen“ Leberversagens.

Pathogenese. Nur etwa $25 \%$ sind akute HBVund HDV-Koinfektionen, die als akutes Leberversagen aufzufassen sind (Mendez et al. 1991).

Akut auf chronische Leberversagen können bei Hepatitis B auch während spontaner Exazerbationen im Verlauf und nach Absetzen einer immunsuppressiven Therapie beobachtet werden (Meyer $u$. Duffy 1993). Das HBV ist nicht direkt zytopathologisch und die Leberschädigung geschieht im Rahmen der immunologischen Antwort auf die Infektion und Elimination befallener Zellen. Demzufolge wird das akute Leberversagen als überschießende Immunantwort auf die HBV-Infektion aufgefaßt (Bernuau et al. 1986). Zum Zeitpunkt der klinischen Manifestation des Leberversagens ist oft bereits kein HBsAg mehr nachweisbar, das HBe-Antigen im Serum ist bereits wieder negativ und nur bei einem kleinen Teil der Patienten ist noch HBV-DNA im Serum nachweisbar (Gimson et al. 1983).

$\mathrm{Ob}$ die HBe-Antigen-negativen "präcore-stopcodon"-Mutanten häufiger als der nichtmutierte HBV-Wildtyp mit einem akuten Leberversagen einhergehen, ist fraglich (Liang et al. 1991).

\section{Hepatitis C}

Das Risiko, nach einer Hepatitis-C-Infektion ein akutes Leberversagen zu entwickeln, ist sehr gering. Genaue Zahlen liegen nicht vor, da bisher keine größeren Serien publiziert wurden. Die früher als "post-Transfusionshepatitis" klassifizierten Fälle, von denen die meisten Hepatitis-C-bedingt waren, zeigten jedoch fast nie ein akutes Leberversagen. 
Einzelfälle von HCV-assoziiertem akutem Leberversagen sind aber berichtet worden (Theilmann et al. 1992).

\section{Kryptogene Hepatitis}

Ein erheblicher Teil der Patienten mit fulminantem Leberversagen zeigt ein Krankheitsbild, welches mit einer Virushepatitis gut vereinbar wäre, bei dem sich jedoch keine der bekannten Virusinfektionen objektivieren läßt. Diese „kryptogene Hepatitis" ist besonders häufig in der Gruppe der protrahierten, subakuten Leberversagen. Hier macht sie in einzelnen Studien bis zu 90 \% der Fälle aus (Gimson 1996).

\section{Hepatitis E}

Bei der in Deutschland extrem seltenen HEV-Infektion werden akute Leberversagen recht häufig beschrieben. Das Risiko soll für Männer bei etwa $3 \%$, bei schwangeren Frauen sogar bei 10-20\% liegen. Besonders groß ist die Sterblichkeit im 3. Trimenon der Schwangerschaft (Irshad 1997).

Ob diese Daten auch außerhalb der Dritten Welt so reproduziert werden, steht noch nicht fest. Die HEV-Infektion scheint aber häufiger zum akuten Leberversagen zu führen als HAV- und HBV-Infektion.

\section{Seltene Ursachen}

Seltene virale Ursachen für ein fulminantes Leberversagen sind Infektionen mit Herpes-simplexViren Typ 1 und 2, Herpesvirus Typ 6, VarizellaZoster-Virus, Eppstein-Barr- und Zytomegalievirus. Auch nach Parainfluenzaviren sind vereinzelt fulminante Hepatitiden beschrieben worden (Williams et al. 1992).

\subsection{2}

\section{Medikamente}

Paracetamol (s. Kap. 45.1.2)

Die Paracetamolüberdosierung wird v.a. in Großbritanien häufig gesehen, jedoch nimmt auch in Deutschland die Zahl der Fälle tendentiell zu. Meist handelt es sich um eine in suizidaler Absicht eingenommene Überdosis.

Die Toxizität ist weitgehend dosisabhängig und vorhersagbar. Oberhalb einer eingenommenen Menge von $10-12 \mathrm{~g}$ ist in der Regel mit deutlicher Lebertoxizität $\mathrm{zu}$ rechnen; gelegentlich beobachtet man jedoch auch Fälle von Leberschädigung bei Einnahme von therapeutischen Mengen, besonders bei Patienten die chronisch Alkohol trinken oder Medikamente einnehmen, die zu einer p450Enzyminduktion führen (Bray et al. 1992).
Pathogenese. Die Metabolisierung des Acetaminophens über das p450-Oxigenasensystem führt zur Bildung eines toxischen, instabilen Metaboliten, der normalerweise durch Konjugation mit Gluthathion schnell inaktiviert wird. Bei Erschöpfung des Gluthathionpools kommt es zur Akkumulation dieses Metaboliten und damit zur Zellschädigung durch Lipidperoxidation (Harrison et al. 1990).

Blutspiegel des Paracetamols 4-16h nach Einnahme können Patienten identifizieren, die möglicherweise eine Lebernekrose erleiden werden.

Therapie. N-Acetylcystein stabilisiert den Gluthathionpool und kann so die Toxizität des Paracetamols erheblich reduzieren. Es muß möglichst früh im Verlauf intravenös verabreicht werden und sollte durchgehalten werden, bis keine Blutspiegel des Paracetamols mehr nachweisbar sind (O'Grady 1997 a).

Verlauf. Das Vollbild des Leberversagens entwikkelt sich verzögert. Typischerweise tritt erst am 3. oder 4. Tag nach Einnahme der Substanz eine sich dann rasch verschlechternde Enzephalopathie ein, gleichzeitig geht die Lebersyntheseleistung zurück und das Bilirubin steigt an (Pereira et al. 1992).

\section{Halothan (s. Kap. 45)}

Die Halothan-Hepatitis ist der Prototyp einer idiosynkratischen Medikamentenreaktionen. Sie kann - wenngleich seltener - auch nach Anästhesie mit anderen Halogen-Kohlenwasserstoffen auftreten (Neuberger 1990).

Typischerweise entwickelt sich schon innerhalb von einer bis 2 Wochen nach Exposition bei den betroffenen Patienten eine rasch progrediente, hochikterische Hepatitis. In der Regel tritt eine Leberschädigung erst nach mindestens 2 facher Halothanexposition auf, in seltenen Fällen kann jedoch schon die erste Exposition zur Hepatitis führen. Betroffen sind typischerweise übergewichtige Frauen im mittleren Lebensalter mit atopischer Diathese. Es handelt sich um eine allergisch-toxische Reaktion bei der halothanspezifische Antikörper beschrieben worden sind (Van Pelt et al. 1995). Diese können aber international nur von wenigen Labors bestimmt werden, so daß die Diagnose in der Regel aufgrund des klinischen Verlaufs gestellt werden muß.

\section{Andere Medikamente}

Idiosynkratische Reaktionen mit Leberversagen können durch eine ganze Reihe von Medikamenten ausgelöst weden (Pohl 1990). Meist tritt die Reaktion in zeitlich kurzem Abstand nach Beginn der Exposition auf. Angesichts der großen Zahl poten- 
tiell verursachender Medikamente ist eine positive Bestätigung der Diagnose oft schwierig, zumal valide Bestätigungsteste fehlen.

Selten können Antikörperphänomene oder klinische Hinweise für andere Hypersensitivitätsreaktionen hilfreich sein. Meist bleibt aber die Diagnose einer idiosynkratischen Medikamentenreaktion eine Ausschlußdiagnose.

\subsection{3 \\ Sonstige Ursachen}

\section{Knollenblätterpilzvergiftungen}

Knollenblätterpilzvergiftungen sind in Zentraleuropa im Spätsommer/Herbst nicht selten. Diagnostisch hilfreich ist der typische Verlauf der Vergiftung (O’Brien 1996).

Klinische Symptome. Innerhalb der ersten 6-12 h nach Ingestion treten schwere abdominelle Schmerzen, Übelkeit und - häufig blutiges - Erbrechen auf. Hiervon erholen sich die Patienten langsam und es folgt eine relativ symptomarme Phase von 1-3 Tagen, während derer sich aber die biochemischen Leberwerte ständig und dramatisch verschlechtern (Koppel 1993).

Kennzeichnend ist ein rascher Abfall der Gerinnungsfaktoren bei steilem Anstieg der Transaminasen. An Tag 3-5 der Vergiftung entwickelt sich dann das Vollbild des akuten Leberversagens, wobei sehr rasch ein Multiorganversagen, insbesondere mit Niereninsuffizienz eintritt.

Diagnostik. In entsprechenden toxikologischen Instituten kann der Nachweis der Amanita-Toxine aus dem Blut die Diagnose sichern und so retrospektiv zur Klärung fraglicher Fälle beitragen.

Therapie. Therapeutisch werden Penicillin G und Silibenin (Mariendistel) verwendet; Studien zur Therapie existieren jedoch praktisch nicht.

\section{Akute Schwangerschaftsfettleber}

Die akute Schwangerschaftsfettleber ist eine Komplikation des letzten Trimenons der Schwangerschaft (s. Kap. 91). Kennzeichnend ist die massive, mikrovesikuläre Verfettung der Leber. Abzugrenzen sind andere schwangerschaftsassoziierte Leberfunktionsstörungen, insbesondere das HELLP(„hypertonus elevated liverenzymes low platelet“-) Syndrom, welches in die Gruppe der schweren Gestosen einzuordnen ist (Riely 1994).

Bei allen schwangerschaftsbezogenen Leberfunktionsstörungen ist, wenn irgend möglich, die sofortige Beendigung der Schwangerschaft anzustreben, danach kommt es in aller Regel zu einer raschen Erholung der Leberfunktion.

\section{Morbus Wilson (s. Kap. 66)}

Ein akutes Leberversagen als Erstmanifestation eines M. Wilson tritt nur bei jungen Menschen, meist im 2. oder 3. Lebensjahrzehnt, auf.

Klinische Symptome. Klinisch sind diese Fälle durch die Trias aus akutem Leberversagen, Coombs-negativer hämolytischer Anämie und diskrepant gering erhöhten Serumenzymwerten, insbesondere für die alkalische Phosphatase, gekennzeichnet (Stremmel et al. 1991). Oft findet sich ein Kayser-Fleischer-Ring.

Histologisch besteht trotz der Präsentation als akutes Leberversagen in diesen Fällen immer schon eine Zirrhose. Die Urin-Kupfer-Ausscheidung ist extrem erhöht und kann neben dem erhöhten Serumkupfer und dem in diesen Fällen fast immer erniedrigten Serumcoeruloplasmin zur Diagnosefindung helfen. Auch das Verhältnis von alkalischer Phosphatase im Serum (U/l) zum Gesamt-Bilirubin $(\mu \mathrm{mol} / \mathrm{l})$ ist als diagnostisches Kriterium vorgeschlagen worden. Ein Wert von weniger als 2 bei Patienten mit fulminantem Leberversagen gilt als suggestiv für das Vorliegen eines M. Wilson (Berman et al. 1991).

\section{Andere Ursachen}

Weitere Ursachen für ein akutes Leberversagen sind - das Budd-Chiari-Syndrom, wenn gleichzeitig alle 3 Lebervenenhauptäste verschlossen sind (Kuo et al. 1996) (s. Kap. 83),

- Hitzeschock, insbesondere nach vorhergegangenem Alkoholexzeß (Feller u. Wilson 1994),

- septische Infektionen,

- kardiovaskuläre Schockzustände,

- das Reye-Syndrom, charakterisiert durch Enzephalopathie, fettige Degeneration der Leber und Leberversagen. Es tritt überwiegend bei Kindern auf, die während eines Virusinfekts (Varizellen, Influenza) Salizylate einnehmen. Seit 1987 ist die Zahl der Erkrankungen bei dieser pathogenetisch nicht eindeutig geklärten Erkrankung deutlich zurückgegangen (Belay et al. 1999).

\section{2 \\ Prognose}

Die Prognose des akuten Leberversagens ist abhängig von der zugrundeliegenden Ätiologie, dem Alter des Patienten und der Dynamik, mit der sich das Krankheitsbild entwickelt (Williams 1996). 
Entwickelt sich das Leberversagen fulminant, d. h. liegen zwischen Ikterus und Enzephalopathie weniger als 7 Tage, so ist die Prognose besser als bei langsamerer Entwicklung. Besonders schlecht ist die Prognose bei subakutem Leberversagen, welches sich über Wochen bis zum Vollbild steigert.

Ätiologisch sind die Medikamententoxizität und die Non-A-Non-B-Non-C-Hepatitis prognostisch schlecht, die Hepatitis A dagegen eher günstig. Schlecht ist die Prognose bei Kindern unter 10 und Patienten über 40 Jahren.

Insgesamt hat sich die Prognose des akuten Leberversagens durch die Weiterentwicklung der Intensivmedizin in den letzten 20 Jahren zwar verbessert, noch immer liegen aber die Überlebensraten nach Eintritt einer Enzephalopathie bei konservativer Therapie allein nur zwischen 30 und $60 \%$ je nach Patient und Diagnose, d. h. etwa jeder zweite Patient mit akutem Leberversagen benötigt eine Lebertransplantation (Williams u. Wendon 1994).
Patienten, die ein akutes Leberversagen überleben, erholen sich in der Regel vollständig. Nur selten bleiben zerebrale, renale oder hepatische Residuen zurück (Karvoutzis et al. 1974).

\section{3}

\section{Klinische Probleme und Therapie (Tab. 46.1)}

\subsection{1 \\ Enzephalopathie (s. Kap. 11)}

Die hepatische Enzephalopathie gehört zu den essentiellen klinischen Befunden für die Diagnose eines akuten Leberversagens. Sie wird eingeteilt in 4 Schweregrade, wobei die Tiefe der Enzephalopathie insbesondere in den Anfangsstadien eines Leberversagens und bei sog. "late onset hepatic failure“ deutlich wechseln kann.

Der Grad der Enzephalopathie hat prognostische Bedeutung. Bleibt der Patient ansprechbar und ori-

Tabelle 46.1. Spezifische Probleme und Therapieansätze beim akuten Leberversagen

\begin{tabular}{|c|c|c|}
\hline Problem & Monitoring & Therapieoption \\
\hline Enzephalopathie & $\begin{array}{l}\text { Gradierung 1-4 } \\
\text { Ansprache } \\
\text { neurologische Untersuchung }\end{array}$ & $\begin{array}{l}\text { NPO } \\
\text { Laktuloseeinläufe } \\
\text { Flumazenil }\end{array}$ \\
\hline Hirnödem & $\begin{array}{l}\text { Systolische RR-Spitzen } \\
\text { intrakranielle Druckmessung } \\
\text { juguläre } \mathrm{O}_{2} \text {-Sättigung }\end{array}$ & $\begin{array}{l}\text { (Hyperventilation) } \\
\text { Mannitol } \\
\text { Thiopental } \\
\text { Luxus-Oxigenierung }\end{array}$ \\
\hline Hyperdynames Kreislaufversagen & $\begin{array}{l}\text { EKG-Monitor } \\
\text { i. a. Druckmessung } \\
\text { Pulmonaliskatheter } \\
\text { Laktat }\end{array}$ & $\begin{array}{l}\text { Katecholamine } \\
\text { ACC (?) } \\
\text { Prostazyklin (?) }\end{array}$ \\
\hline Nierenversagen & $\begin{array}{l}\text { Urinmenge } \\
\text { Urin-Natrium } \\
\text { Serumkreatinin }\end{array}$ & $\begin{array}{l}\text { Hydratation optimieren } \\
\text { Low-dose-Dopamin } \\
\text { CAVH/CVVH }\end{array}$ \\
\hline Hepato-pulmonales Syndrom & $\begin{array}{l}\mathrm{paO}_{2} / \mathrm{paCO}_{2} \\
\text { elektive Intubation } \\
\text { Pulmonaliskatheter }\end{array}$ & $\begin{array}{l}\text { Cave Uberwässerung! } \\
\text { hohes } \mathrm{fiO}_{2} \\
\text { niedriger intrathorakaler Druck }\end{array}$ \\
\hline Gerinnungsstörungen & $\begin{array}{l}\text { Prothrombinzeit (Quick) } \\
\text { Faktor II, V, AT III } \\
\text { Fibrinspaltprodukte } \\
\text { Thrombozyten }\end{array}$ & $\begin{array}{l}\text { Fresh frozen plasma (Soll }>20 \% \text { ) } \\
\text { AT-III-Substitution auf }>50 \% \\
\text { Low-dose-Heparin } \\
\text { Blutungsprophylaxe mit PPI }\end{array}$ \\
\hline Metabolische Entgleisung & $\begin{array}{l}\text { BZ-Kontrollen } \\
\mathrm{Na}^{+}, \mathrm{K}^{+}, \mathrm{pH}, \mathrm{HCO}^{-} \\
\text {Harnstoff }\end{array}$ & $\begin{array}{l}\text { Glukosedauerinfusion } \\
\text { i. v. Ernährung }\end{array}$ \\
\hline Sepsis & $\begin{array}{l}\text { Mikrobiologische Kulturen } \\
\text { Abstriche } \\
\text { CRP } \\
\text { Procalcitonin }\end{array}$ & $\begin{array}{l}\text { Prophylaktische SDD } \\
\text { nach Intubation: prophylaktische Antibiose }\end{array}$ \\
\hline
\end{tabular}

NPO „nichts per os“; keine orale Nahrungszufuhr;

ACC Acetylcystein;

CAVH kontinuierliche arteriovenöse Hämofiltration;

$C V V H$ kontinuierliche venovenöse Hämofiltration;

$S D D \quad$ selektive enterale Darmdekontamination. 
entiert (Enzephalopathiegrade 1 und 2), so ist die Prognose gut; tritt jedoch eine höhergradige Enzephalopathie ein, wird die Prognose generell deutlich schlechter und unvorhersagbarer (Ellis u. Wendon 1996). Das Risiko eines Multiorganversagens und die Gefahr eines Hirnödems steigen parallel zum Grad der Enzephalopathie an.

\section{Pathophysiologie}

Die Pathophysiologie der Enzephalopathie ist multifaktoriell und nicht vollständig geklärt. Funktionell kommt es zu einer Verschlechterung des neuronalen Energiestoffwechsels und einer Veränderung der Blut-Hirn-Schranke. Ammoniak, Phenole, Fettsäuren, Mercaptane und sog. Mittelmoleküle sind alle als verursachende Substanzen angeschuldigt worden.

Gammaaminobuttersäure (GABA). Neuerdings wurde auch die Bedeutung der GABA für die „endogene Narkose“ der hepatischen Enzephalopathie untersucht. Bei dieser Substanz handelt es sich um den wesentlichsten inhibitorischen Neurotransmitter im ZNS. Sein Wirkungsprofil läßt eine Vermittlung über den GABA-Rezeptor als möglich erscheinen (Jones et al. 1984). Die zirkulierenden GABA-Spiegel bei Patienten mit akutem Leberversagen sind erhöht gefunden worden. Andererseits sind die GABA-Konzentrationen im Hirngewebe und im Liquor bei Patienten mit schwerer Enzephalopathie meist normal.

Alternativ sind Mechanismen untersucht worden, die die Empfindlichkeit des GABA-Rezeptors steigern könnten, insbesondere der Benzodiazepinrezeptor, der mit dem GABA-Rezeptor in der Plasmamembran neuronaler Zellen einen supramolekularen Komplex bildet. Ein endogener Benzodiazepinligant mit einem Molekulargewicht von etwa 10.000 ist im Liquor von Patienten mit hepatischer Enzephalopathie und bei Patienten, die an einem akuten Leberversagen verstarben, beschrieben worden (Jones et al. 1990). Konsequenterweise wurde versucht, durch Gabe von Benzodiazepinantagonisten die hepatische Enzephalopathie zu verhindern.

Die Erfolge einer solchen Therapie sind zwar in einzelnen Fällen eindrucksvoll, jedoch stets nur kurzfristig und so variabel, daß die Stimulation des GABA-Rezeptors nicht die alleinige Erklärung für die Enzephalopathie sein kann (Gyr u. Meier 1991).

Ebenfalls diskutiert wurden als mögliche Ursache die Bildung "falscher Neurotransmitter" im Serum und im ZNS sowie die Veränderungen des zirkulierenden Aminosäureprofils. Letztlich ist die Genese der hepatischen Enzephalopathie ungeklärt, gesichert ist aber, daß die Aufnahme stickstoffhaltiger Substanzen aus dem Darm die Symptomatik verschlechtert. Eiweißkarenz und hohe Einläufe mit Laktulose sind deshalb insbesondere in frühen Enzephalopathiestadien empfehlenswert.

\subsection{2}

\section{Hirnödem}

Das Hirnödem ist eine der häufigsten Todesursachen bei akutem Leberversagen. 75-80\% der Patienten mit Enzephalopathie Grad 4 entwickeln ein Hirnödem, unabhängig von der dem Leberversagen zugrundeliegenden Ursache (Blei 1995). Jüngere Patienten sind besonders gefährdet, und die Gefahr ist besonders groß bei fulminantem Verlauf. Bei subakutem Leberversagen wird ein Hirnödem selten beobachtet.

\section{Klinische Symptome}

Klinisch findet sich bei Patienten, die ein Hirnödem entwickeln, initial ein systolischer Blutdruckanstieg, eine Hyperventilation, träge Pupillenreaktion und schließlich ein Ausfall der Hirnstammreflexe.

Klassische Stauungspapillen sieht man selten. Eine direkte Messung des intrakraniellen Drucks muß angestrebt werden, wird jedoch durch die ausgeprägte Gerinnungsstörung im Verlauf immer schwerer möglich. Patienten, die eine zunehmende Enzephalopathie entwickeln, sollten darum möglichst frühzeitig eine peridurale Hirndrucksonde erhalten (Cordoba u. Blei 1995).

\section{Hirndruckmessung}

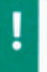

Bei beatmeten Patienten ist die direkte Druckmessung besonders wünschenswert, da klinische Hirndruckzeichen bei ihnen kaum noch evaluierbar sind. Außerdem ermöglicht die direkte Druckmessung die bessere Führung der Patienten während diagnostischer oder therapeutischer Eingriffe, die den Hirndruck erhöhen können, wie z. B. Bronchoskopie oder Hämodialyse.

Bei einer evtl. nachfolgenden Transplantation ist die Hirndruckmessung von großem Vorteil, da es im Verlauf der Operation zu erheblichen Schwankungen des zerebralen Perfusionsdrucks kommen kann (Keays et al. 1991).

Die Komplikationsrate der periduralen intrakraniellen Druckmessung liegt insgesamt bei etwa $4 \%$ bei Verwendung moderner Drucksonden (Blei et al. 1993). 
Perfusionsdruck. Die Bedeutung der zerebralen Druckerhöhung liegt in ihren negativen Folgen für die Hirndurchblutung. Der zerebrale Perfusionsdruck errechnet sich als Differenz zwischen dem Hirndruck und dem arteriellen Mitteldruck, so daß bei steigendem Hirndruck und fallendem arteriellem Mitteldruck der zerebrale Perfusionsdruck rasch abnimmt (Munoz et al. 1993). Kritisch sind Werte unter $50 \mathrm{mmHg}$; länger anhaltende Werte unter $40 \mathrm{mmHg}$ werden nur selten überlebt (Aggarwal et al. 1994).

Verfahrensweise. Patienten, die in Gefahr sind ein Hirnödem $\mathrm{zu}$ entwickeln, sollten in ruhiger Umgebung gepflegt werden, der Oberkörper soll $45 \mathrm{Grad}$ angehoben gelagert werden, und direkte Manipulationen am Patienten sollen auf ein Minimum beschränkt bleiben. Patienten mit Enzephalopathie Grad 3 oder 4 sollten elektiv intubiert werden, um zu verhindern, daß eine Aspiration entritt und um eine maschinelle Hyperventilation $\mathrm{zu}$ ermöglichen.

Ein Monitoring des Sauerstoffgehalts in der V. jugularis im Vergleich zur arteriellen Sauerstoffsättigung ermöglicht eine Abschätzung des zerebralen Sauerstoffverbrauchs (Larsen et al. 1996). Dieser sollte im Verlauf erfaßt werden, um eine Verschlechterung frühzeitig erkennen und therapeutisch reagieren zu können. Auch ein Anstieg des jugulären Laktatspiegels deutet auf eine ungenügende Sauerstoffversorgung des Gehirns hin.

\section{Verlauf}

In frühen Stadien des Hirnödems ist die intrakranielle Druckerhöhung schwankend und der zerebrale Blutfluß erhöht. Die Sauerstoffaufnahme des Gehirns ist noch normal.

Mit fortschreitendem Ödem wird die Druckerhöhung stärker und zeigt keine Schwankungen mehr. Nunmehr sinkt der zerebrale Perfusionsdruck; in der Folge kommt es zu einer Abnahme des Blutflusses und zu einer Verminderung der Sauerstoffzufuhr. In diesen Stadien steigt die arteriell/juguläre Sauerstoffdifferenz deutlich an, da die zerebrale Sauerstoffextraktion steigt, um den verminderten Blutfluß zu kompensieren.

In Spätstadien reicht die Hirndurchblutung nicht mehr für eine Versorgung der Nervenzellen aus, und es kommt zum Hirntod (Wijdicks et al. 1995). Dieser tritt u. U. auch ohne Zeichen einer zerebellären Einklemmung auf.

\section{Therapie}

Hyperventilation. Therapeutisch ist die Hyperventilation auf $\mathrm{paCO}_{2}$-Werte zwischen 30 und 35 nur in Frühstadien erfolgversprechend. Nimmt mit fortschreitender Druckerhöhung der zerebrale Blutfluß ohnehin ab, so ist keine Hyperventilation mehr angezeigt.

Mannitol. Mannitol zur osmotischen Hirnödemtherapie ist das wichtigste Therapieprinzip. Es ist besonders in frühen und mittleren Stadien des Hirnödems wirksam, verliert in späten Stadien jedoch deutlich an Effizienz (Williams u. Gimson 1991). Die Wirkung des Mannitol geht mit einer osmotischen Diurese einher. Zeigen Patienten ohne Nierenversagen nach Gabe von 0,3-0,4 mg/kg Mannitol keine Zunahme der Diurese, soll die Plasmaosmolarität gemessen werden. Liegt sie unter 320 mOsm, soll die Dosis unmittelbar wiederholt werden. Bei Patienten mit Nierenversagen ist Mannitol nur wirksam, wenn innerhalb von 15 min nach Administration das 3 fache des zugeführten Volumens durch Ultrafiltration entfernt wird.

Weitere Maßnahmen. In späten Stadien sind eine Verminderung des Sauerstoffbedarfs durch Gabe von Thiopental und eine Verbesserung des Sauerstoffangebots durch Erhöhung des inspiratorischen Sauerstoffs („Luxus-Oxygenierung“) die einzig verbleibenden Therapieansätze (Lee 1996). Eine Überwässerung der Patienten mit fulminantem Leberversagen muß durch sorgfältige Volumenüberwachung und Flüssigkeitsbilanzierung verhindert werden.

Fällt der zerebrale Perfusionsdruck unter $50 \mathrm{mmHg}$, muß durch Katecholamine der arterielle Mitteldruck angehoben werden. In diesem Stadium sollte der Patient flach gelagert werden, um den zerebralen Blutfluß zu optimieren. Die Gabe von Dexamethason bei hepatisch induziertem Hirnödem ist nicht hilfreich (Davies et al. 1994).

\subsection{3 \\ Kardiovaskuläres System und Hämodynamik}

\section{Körperliche Symptome}

Systolische Blutdruckerhöhungen bei Patienten mit Grad-4-Enzephalopathie sind Hinweise für eine intrakranielle Druckerhöhung. Typischerweise findet sich im akutem Leberversagen dagegen ein hypotoner, hyperdynamer Kreislauf, wie bei Patienten mit Sepsis. Die Situation wird gekennzeichnet durch ein hohes Herz-Zeit-Volumen bei deutlich erniedrigtem peripheren Widerstand sowie niedrigen diastolischen Blutdruckwerten (Ellis u. Wendon 1996).

Relative Hypovolämie durch Vasodilatation führt $\mathrm{zu}$ niedrigen zentralvenösen, pulmonalarteriellen und linksventrikulären Füllungsdrucken. 


\section{Volumenersatz}

Die Patienten mit Grad-4-Enzephalopathie und hohem Hirndruckrisiko müssen mit einem Pulmonaliskatheter überwacht werden, um sie optimal hydrieren und die peripheren Widerstände mit Katecholaminen präzise einstellen zu können. Flüssigkeitsersatz soll immer auch mit Blutkonserven erfolgen, wenn der Hämoglobinwert unter $11 \mathrm{~g} \%$ absinkt. Persistiert das hypotone Kreislaufversagen trotz adäquater Hydratation, so ist die Prognose sehr schlecht.

\section{Katecholamine}

Katecholamine können die Situation stabilisieren. Ihr Einsatz ist aber nur mit einer Verbesserung der Prognose verbunden, wenn weiter therapeutische Maßnahmen ergriffen werden.

Adrenalin und Noradrenalin verbessern die Kreislaufsituation durch Steigerung des peripheren Widerstands. Adrenalin unterstützt darüber hinaus die myokardiale Kontraktionskraft. Keine der beiden Substanzen erhöht aber die Sauerstoffaufnahme in peripheren Geweben.

\section{Supportive Maßnahmen}

Zusätzliche Maßnahmen, die das mikrovaskuläre Sauerstoffangebot und die periphere Sauerstoffaufnahme verbessern, werden insbesondere von angelsächsischen Arbeitsgruppen sehr empfohlen. Diese konnten zeigen, daß die intravenöse Gabe von Prostacyclin die Mikrozirkulation und den Sauerstoffverbrauch in der Peripherie verbessert (Wendon et al. 1992) und eine Dauerinfusion von $\mathrm{N}$-Acetylcystein, in Dosen wie bei Paracetamolintoxikation, die Sauerstoffextraktion und den Sauerstoffverbrauch steigern kann (Devlin et al. 1997).

Insgesamt sollen als Zielgrößen der hämodynamischen Maßnahmen folgende Richtwerte erreicht werden:

- ein Herzindex von über $4,5 \mathrm{l} / \mathrm{min} / \mathrm{m}^{2}$,

- ein peripherer Widerstandsindex von über $700 \mathrm{dyn} / \mathrm{s} / \mathrm{cm}^{-5}$ und

- ein $\mathrm{O}_{2}$-Verbrauch von über $170 \mathrm{ml} / \mathrm{min} / \mathrm{m}^{2}$.

\subsection{4}

\section{Nierenversagen}

Zwischen 30 und $75 \%$ der Patienten mit akutem Leberversagen entwickeln ein Nierenversagen mit Oligoanurie und Kreatininanstieg (Mendoza et al. 1997).

Bei Paracetamol- und Knollenblätterpilzvergiftung ist das Nierenversagen ein direkt toxischer Effekt der zugrundeliegenden Intoxikation und ent- wickelt sich häufig schon vor Eintritt der Enzephalopathie.

Patienten mit Leberversagen anderer Ursache zeigen typischerweise bei fortgeschrittener Enzephalopathie zunächst ein funktionelles Nierenversagen mit Abfall des Urinnatriums auf unter $10 \mathrm{mmol} / \mathrm{l} \mathrm{mit}$ anschließendem Tubulusschaden und Anurie (Davison 1996).

Ein verminderter intravasaler Druck durch Hypovolämie oder Vasodilatation trägt zur Verschlechterung der Nierenfunktion bei und muß ausgeglichen werden. Das Nierenversagen bei Leberausfall ist gekennzeichnet durch eine extreme intrarenale Vasokonstriktion. Diese ist lange Zeit reversibel und führt erst nach protrahiertem Verlauf zu bleibenden Schäden an der Niere.

\section{Medikamentöse Therapie}

Dopamin in niedriger Dosis $(2-4 \mu \mathrm{g} / \mathrm{kg} / \mathrm{h}) \mathrm{kann}$ möglicherweise in Frühstadien die Verschlechterung der Nierenfunktion durch eine Verbesserung des renalen Blutflusses verzögern, solange die Patienten noch nicht anurisch sind.

Hohe Dosen Furosemid sind wirkungslos und nicht empfehlenswert, andere nephrotoxische Medikamente wie Aminoglykoside, Vancomycin und auch Mannitol sollen nur mit strenger Indikation zur Anwendung kommen (Munoz 1993).

\section{Dialyse}

Nierenersatztherapie soll frühzeitig, noch vor Erreichen der sonst üblichen Dialysekriterien begonnen werden, um Überwässerungen mit Lungenödem und der vermehrten Gefahr eines Hirnödems zu vermeiden. Die Patienten sind häufig kreislaufinstabil, daher sind kontinuierliche Hämofiltrationsverfahren gegenüber intermittierender Dialyse vorzuziehen.

\section{Prognose}

Die Prognose des akuten Leberversagens wird durch das zusätzliche Nierenversagen erheblich verschlechtert. Nur $30 \%$ der Patienten mit fulminanter Hepatitis A oder B überleben diese Befundkonstellation.

\subsection{5 \\ Pulmonale Komplikationen}

Mit höhergradiger Enzephalopathie und insbesondere bei beginnender Druckerhöhung zeigen viele Patienten eine deutliche arterielle Hypoxämie (Bihari et al. 1986), die unter Beatmung ein hohes Sauerstoffangebot notwendig macht. 


\section{Ursachen}

Die Ursachen hierfür sind vielfältig. Patienten mit Leberversagen haben häufig bronchopulmonale Infektionen. Bei der Hälfte lassen sich pathogene Keime im Trachealsekret finden und etwa ein Viertel der Patienten zeigt bronchopneumonische Infiltrate im Röntgenbild (Wyke 1989). Häufig entwikkelt sich zudem ein pulmonales Kapillarleck mit nichtkardialem Lungenödem und ARDS-ähnlichem Bild, insbesondere wenn große Mengen Albumin, Gerinnungsfaktoren und Frischplasma substituiert werden müssen.

Unabhängig von diesen eher unspezifischen pulmonalen Veränderungen beobachtet man bei einem Teil der Patienten darüber hinaus einen deutlichen Ventilations/Perfusions-Mismatch. Die Situation erinnert an das bei chronischen Lebererkrankungen beobachtete "hepatopulmonale Syndrom" (Krowka 1993) und ist gekennzeichnet durch eine erhebliche intrapulmonale Vasodilatation, die $\mathrm{zu}$ einem funktionellen Rechts-Links-Shunt führt.

\section{Therapie}

Hohe Sauerstoffpartialdrucke sind erforderlich, um eine ausreichende Oxigenierung des Blutes sicherzustellen. Bei der Beatmung dieser Patienten soll dennoch der Atemwegsmitteldruck nicht wesentlich gesteigert werden, um nicht durch Stauung des venösen Rückflusses die Entwicklung eines Hirnödems zu begünstigen.

\subsection{6 \\ Gerinnungsstörung}

Schwere Gerinnungsstörungen gehören obligat zum klinischen Bild des akuten Leberversagens. Die Natur dieser Gerinnungsstörungen ist komplex und umfaßt sowohl einen Mangel an prokoagulatorischen Faktoren als auch ein Defizit bei den Inhibitoren der Gerinnung und der Fibrinolyse (Pereira et al. 1996).

Man findet bei akutem Leberversagen einen Mangel an Fibrinogen, Prothrombin, Faktor II, V, VII, IX Protein S, Protein C, und Antithrombin III.

Quick. Die Prothrombinzeit („Quick-Wert“) wird häufig als aussagekräftiger Indikator für die Schwere des Leberversagens benutzt (Anand et al. 1997). Sie mißt hauptsächlich die Faktoren II, V, VII und $\mathrm{X}$.

Neben einer verminderten Produktion durch die Leber spielt auch ein gesteigerter peripherer Verbrauch an Gerinnungsfaktoren für die niedrigen Spiegel eine Rolle. Zwar sieht man nur selten das Vollbild der Verbrauchskoagulopathie, bei Anwen- dung sensitiver Suchtests zeigen jedoch fast alle Patienten Zeichen der disserminierten intravasalen Gerinnung (Langley et al. 1990).

Antithrombin III. Antithrombin-III-Mangel findet sich bei fast allen Patienten. Die Substitution auf Werte über $50 \%$ ist assoziiert mit einer längeren Halbwertszeit für Heparin und einer geringeren Koagulopathierate insbesondere bei Patienten die dialysiert oder hämofiltriert werden müssen (Langley et al. 1991).

Thrombozyten. Ebenfalls gut dokumentiert sind qualitative und quantitative Störungen der Thrombozyten. Mehr als 2 Drittel der Patienten haben zirkulierende Thrombozytenzahlen unter $100.000 / \mu l$. Die Thrombozytenaggregation ist gestört, jedoch findet sich eine gesteigerte Adhäsionsbereitschaft der Thrombozyten möglicherweise als Folge gesteigerter Willebrand-Faktor-Spiegel (Langley et al. 1985).

\section{Therapie}

In frühen Untersuchungen wurden klinisch schwere Blutungen bei $30 \%$ der Patienten beschrieben. Seither hat die großzügige Substitution mit gefrorenem Frischplasma diese Rate deutlich vermindert.

Die häufigste Blutungsquelle mit relevantem Blutverlust ist die Schleimhaut im Magen und oberen Dünndarm (Ellison et al. 1996). Dies ist Folge der akuten Stauungsgastritis mit häufig ausgedehnten Erosionen der Magenschleimhaut. Patienten im akuten Leberversagen müssen daher unbedingt $\mathrm{H}_{2}$-Blocker oder Protonenpumpeninhibitoren zur Blutungsprophylaxe erhalten.

\subsection{7 \\ Metabolische Störungen}

Glukose. Patienten mit akutem Leberversagen entwickeln häufig eine schwere Hypoglykämie. Die Hypoglykämie kann die mentale Situation der Patienten erheblich verschlechtern und wird gelegentlich als progrediente Enzephalopathie verkannt (Lee 1996). Umgekehrt werden duch eine fortgeschrittene Enzephalopathie die Zeichen der Hypoglykämie maskiert, weshalb engmaschige Blutzukkerkontrollen notwendig sind. Zeigen sich Unterzuckerungstendenzen (BZ unter $3,5 \mathrm{mmol} / \mathrm{l}=$ $60 \mathrm{mg} \%)$, muß intravenös Glukose substituiert werden.

Die Ursachen der Hypoglykämieneigung sind vielfältig. Mit fortschreitender Verschlechterung 
der Leberfunktion kommt es zu einem Versagen der Glukoneogenese. Gleichzeitig sind die Patienten meist schon länger ohne adäquate Nahrungsaufnahme, wodurch die Glykogenspeicher leer sind (Barber u. Teasley 1984). Darüber hinaus zeigen viele der Patienten eine Hyperinsulinämie durch verminderte hepatische Insulinextraktion (Fiaccadori et al. 1991).

Säure-Basen-Haushalt. Störungen des SäureBasen Haushalts sind ebenfalls häufig. Bei Paracetamolintoxikation entwickeln bis zu $30 \%$ der Patienten eine metabolische Azidose. Diese geht der Enzephalopathie zeitlich voraus und ist von der Nierenfunktion unabhängig (O'Grady $1997 \mathrm{~b}$ ). Sie verschlechtert die Prognose erheblich. Das Absinken des arteriellen $\mathrm{pH}$-Wertes auf unter 7,3 am zweiten Tag nach Einnahme oder später ist mit einer Mortalität von $90 \%$ assoziiert.

Laktatazidose. Demgegenüber beobachtet man bei nur $5 \%$ der Patienten mit akutem Leberversagen anderer Genese eine Azidose, die ebenfalls mit einer sehr schlechten Prognose assoziiert ist.

Hier handelt es sich meist um Laktatazidosen, bedingt durch mangelnde Sauerstoffaufnahme in der Peripherie (Pandha 1992). Die Ursache liegt in Mikrozirkulationsstörungen durch Tonusveränderungen der Arteriolen, Mikrothromben bei intravasaler Gerinnungsneigung und dem Gewebsödem bei erhöhter Kapillarpermeabilität. Maßnahmen gegen die Laktatazidose, wie Hämodialyse und Bikarbonatsubstitution bleiben wirkungslos, solange nicht die zugrundeliegende Mikrozirkulationsstörung behoben werden kann.

Öfter als schwere Azidosen sieht man bei akutem Leberversagen eine metabolische Alkalose. Diese wird bedingt durch ein Versagen der Harnstoffsynthese in der Leber, wodurch die beiden Vorläufersubstrate der Harnstoffbildung, Ammonium und Bikarbonat, akkumulieren (Häussinger u. Gerok 1986).

In Assoziation mit der Alkalose wird gehäuft eine Hypokaliämie gesehen.

\subsection{8 \\ Ernährung bei Leberversagen}

Die wenigsten Patienten mit akutem Leberversagen sind schon vor ihrer Erkrankung mangelernährt. Mit Verschlechterung der Leberfunktion kommt es jedoch in fast allen Fällen zur Ausbildung einer katabolen Stoffwechsellage, und die Patienten verlieren rasch an Körpermasse.
Glucoseinfusionen sind notwendig zur Verhinderung von Unterzuckerungszuständen. Eine intravenöse Ernährung ausschließlich mit Glukose führt aber schnell zu einer schweren Verfettung des funktionsfähigen Restparenchyms der Leber (Barber u. Teasley 1984).

\section{Enterale Ernährung}

Wenn ausreichend Kalorien angeboten werden sollen, steht man daher vor einer Reihe praktischer Probleme. Enterale Sondenernährung, obgleich für die Leberregeneration wegen der portalen Anflutung der Substrate wünschenswert, läßt sich wegen Darmatonie und der Gefahr einer Verschlechterung der Enzephalopathie durch das Einbringen von Protein in den Darm nicht umsetzen.

\section{Parenterale Ernährung}

Bei intravenöser Ernährung ist man durch die damit u. U. einhergehende Volumenbelastung limitiert. Lange Zeit hat man sich gescheut, Patienten im Leberversagen intravenöse Fette anzubieten. Neuere Ergebnisse zeigen jedoch, daß eine parenterale Ernährung mit Lipiden und Aminosäuren in der Lage ist, die negative Stickstoffbilanz der Patienten zu korrigieren. Ob hierbei mit verzweigtkettigen Aminosäuren angereicherte Lösungen einen Vorteil über Standardmischungen haben, ist immer noch unklar (Alexander et al. 1989).

Substitution wasserlöslicher Vitamine ist sinnvoll. Ob fettlösliche Vitamine und Spurenelemente substituiert werden müssen, ist fraglich. Dennoch neigen die meisten Zentren zu einer Gabe zumindestens von Vitamin $\mathrm{K}$.

\subsection{9}

Sepsis

Patienten mit fulminantem Leberversagen haben eine schwerwiegende Störung ihrer Immunabwehr, mit gestörter Neutrophilen- und KupfferZell-Funktion, sowie einem Mangel an Opsoninen (Komplementfaktoren, Fibronektin; Acharya et al. 1995).

Bei Patienten mit Enzephalopathie von Grad 2 und schwerer finden sich pathologische mikrobiologische Kulturbefunde in $80 \%$ der Fälle. Am häufigsten sind Infektionen der Atemwege (62\%), gefolgt von positiven Urin ( $48 \%$ ) und Blutkulturbefunden $(20 \%)$. Die häufigsten gefundenen Erreger sind Staphylokokken, Streptokokken und coliforme Bakterien. Darüber hinaus zeigen $32 \%$ der Patienten eine Pilzinfektion, meistens mit Candidaarten, die meist später im Verlauf auftritt (Rolando et al. 1992). 
!.

Die Gefahr für Infektionen ist besonders groß bei gleichzeitigem Nierenversagen und maschineller Beatmung. Tägliche Kulturen aller erreichbaren Körperhöhlen und Flüssigkeiten sind unbedingt notwendig, um jederzeit über die aktuelle Keimsituation und die Resistenzlage informiert zu sein.

\section{Medikamentöse Therapie}

Das klinische Bild des akuten Leberversagens weist zahlreiche Übereinstimmungen mit dem der Sepsis auf. Insbesondere das hyperdyname Kreislaufversagen und die latente Verbrauchskoagulopathie lassen die Abgrenzung von gramnegativen Infektionen häufig schwierig werden. Infektionen sind neben dem Hirnödem die häufigste Todesursache bei Patienten mit akutem Leberversagen. Es ist daher immer wieder versucht worden, die Prognose des akuten Leberversagens durch prophylaktische Gabe von Antibiotika und Antimykotika zu verbessern. Dabei konnte gezeigt werden, daß eine Kombination aus intravenösen Breitspektrumantibiotika und oralen Antimykotika die Prognose der Patienten signifikant verbessern kann (Rolando et al. 1992), insbesondere dann, wenn sie später eine Lebertransplantation brauchen. Dabei kommt der prophylaktischen Antimykotikagabe im Rahmen einer sog. selektiven Darmdekontamination die größte Bedeutung zu (Tabelle 46.1).

\section{4}

\section{Spezifische Therapien}

Schwangerschaftsassoziiertes Leberversagen. Ein Schwangerschaftstest soll bei allen weiblichen Patienten mit Leberversagen unmittelbar durchgeführt werden. Handelt es sich um ein schwangerschaftsassoziiertes Leberversagen, muß wenn irgend möglich die Schwangerschaft beendet werden, womit die Leberfunktion in aller Regel kurzfristig normalisiert wird (Baez u. Gonzalez 1994).

Intoxikation. Antidote stehen für die Paracetamolintoxikation und möglicherweise für die Vergiftung mit Amanitatoxinen zur Verfügung.

Bei Paracetamolintoxikation ist die frühzeitige, hochdosierte Gabe von Acetylcystein u. U. lebensrettend und muß solange durchgehalten werden, bis keine Metabolite der Substanz mehr nachweisbar sind. Möglicherweise profitieren die Patienten sogar von einer Gabe der Substanz bis zur Erholung der Leberfunktion (Janes u. Routledge 1992). Bei Knollenblätterpilzintoxikation wird Legalon (Mari- endistel, Silibenin) gegeben, obwohl die Wirksamkeit strenggenommen nicht erwiesen ist.

Darüber hinaus sind spezifische Therapien des akuten Leberversagens nicht als wirksam gesichert. Insulin und Glukagondauerinfusionen wurden eingesetzt, um die Leberregeneration zu fördern. Die Ergebnisse zeigten jedoch keine überzeugenden Effekte und die Gefahr der Blutzuckerentgleisung ist hoch (Woolf u. Redeker 1991).

\section{5}

\section{Prognostische Faktoren}

Untersuchungen der zirkulierenden „hepatic growth factor"-(HGF-)Spiegel zeigen, daß dieser leberspezifische Wachstumsfaktor bei Patienten mit Leberversagen in fast allen Fällen deutlich erhöht ist. Patienten, die starben oder transplantiert werden mußten, zeigten höhere Werte als solche, die sich erholten (Hughes et al. 1994).

Andererseits zeigen Studien, daß eine Erhöhung des Alphafetoproteins im Verlauf des akuten Leberversagens mit einer positiven Prognose assoziiert ist (Jain et al. 1995). Demnach ist die Situation im akuten Leberversagen durch maximale Regenerationsstimulation gekennzeichnet. Das Überleben der Patienten hängt jedoch von der tatsächlich eintretenden Hepatozytenproliferation ab, die wiederum entscheidend vom Ausmaß der zugrundeliegenden Schädigung bestimmt wird. Zukünftige Ansätze werden daher darauf gerichtet sein müssen, die Pathomechanismen der zugrundeliegenden Krankheiten zu charakterisieren und Schädigungsmechanismen frühzeitig zu unterbrechen.

Hierzu unternommene Versuche haben bisher leider nicht überzeugt. Interferon verbessert nicht das Überleben bei fulminanter Virushepatitis (Sanchez-Tapias et al. 1987), und der Versuch, viral induzierte fulminante Hepatitiden durch intravenöses Prostaglandin E1 zu behandeln, blieb ohne reproduzierbaren Erfolg (Cattral et al. 1994).

\section{6 \\ Leberersatzverfahren}

Bei der Entwicklung maschineller Leberersatzverfahren werden 2 grundsätzliche Ansätze verfolgt, nämlich Filtrationsverfahren und zellgestützte Bioreaktoren. 


\subsection{1}

\section{Filtrationsverfahren}

Auf der einen Seite stehen ein Reihe von Filtrationsverfahren. Die Grundkonzeption ist hierbei, daß die Patienten mit akutem Leberversagen letztlich an der fehlenden Entgiftungsfunktion der Leber versterben, also an einer endogenen Vergiftung, die durch Filtration und Entfernung der relevanten Toxine verhinderbar sein müßte (Malchesky 1994).

Ursprünglich wurden hierzu Plasmapheresen durchgeführt, später wurden Kohlefilter und Adsorberharze verwendet. Neueste Maschinen benutzen eine Kombination aus Plasmapherese und Spezialadsorbern. Die Ergebnisse zahlreicher publizierter Studien zeigen, daß die Effekte der verschiedenen Filtrationsverfahren jeweils nur passager und insgesamt enttäuschend sind. Zwar bessert sich häufig besonders bei den ersten Behandlungen - der Bewußtseinszustand der Patienten unter der Filtration. Nach Ende der Behandlung kommt es jedoch unmittelbar $\mathrm{zu}$ einer erneuten Verschlechterung auf das Ausgangsniveau, und eine Verbesserung der Gesamtprognose konnte nie gezeigt werden (McGuire et al. 1995).

Ein wesentliches Handikap bei der Entwicklung effektiver Filtrationssysteme ist, daß bis heute unklar ist, welche Substanzen entfernt werden müßten und welche möglicherweise protektiv und für das Überleben essentiell sein könnten. Daher ist bis heute nicht einmal klar, ob nicht eine konsequente Filtration letzendlich die Prognose sogar verschlechtern würde, indem sie z. B. kompensatorisch erhöhte Regenerationsfaktoren absenkt.

\subsection{2}

\section{Bioreaktoren}

Seit Jahren werden Versuche unternommen, Zellkultursysteme sog. „Bioreaktoren“ zu entwickeln, die zumindest zeitweise die Leberfunktion übernehmen könnten, bis eine Restitution der eigenen Leber erfolgt oder ein geeignetes Transplantat verfügbar wird. Auch bei diesen Ansätzen sind bisher die grundlegenden Probleme ungelöst (Jauregui et al. 1996).

- Reaktoren mit humanen Leberzellkulturen sind von fraglichem Wert, da man praktisch eine humane Leber opfern muß, um den Bioreaktor zu beladen. Es fragt sich, ob es nicht sinnvoller wäre, diese dann gleich zu transplantieren.

- Bei Verwendung von Schweinehepatozyten ist die Biokompatibilität unklar.

- Werden andererseits immortalisierte humane Leberzellinien verwendet, muß deren differen- zierte Funktionstüchtigkeit nachgewiesen und eine Verschleppung der potentiell malignen Zellen in den Organismus des Patienten verhindert werden.

Somit steht bis heute kein anwendungsfähiger Bioreaktor zur Verfügung. Die Forschung auf dem Gebiet der Leberersatzverfahren ist jedoch aktiv, und es erscheint als möglich, daß in den nächsten Jahren funktionsfähige Konzepte umgesetzt und zur klinischen Erprobung gebracht werden.

\section{7 \\ Lebertransplantation}

Die Lebertransplantation hat von allen Therapieformen des akuten Leberversagens für sich genommen die größte Verbesserung der Prognose bewirkt. Die 5-Jahres-Überlebensraten liegen zwar mit knapp $60 \%$ unter denen bei elektiver Transplantation für chronische Leberkrankheiten (Bismuth et al. 1996), stellen aber gegenüber der infausten Spontanprognose der Patienten eine enorme Verbesserung dar.

Die hohe postoperative Todesrate ist Ausdruck der großen Häufigkeit septischer Infektionen bei den Patienten. Diese hängt von der Dauer des Aufenthalts auf der Intensivstation, der Notwendigkeit maschineller Beatmung und dem Ausmaß des Multiorganversagens (Dialyse etc.) ab (McCashland et al. 1996).

Die Indikationsstellung zur Transplantation bei akutem Leberversagen muß unter Berücksichtigung der Ätiologie und des Verlaufs erfolgen. Grundsätzlich soll jede Chance zu einer Erholung der Leberfunktion ohne Transplantation genutzt werden, da in Fällen, in denen ein akutes Leberversagen überlebt wird, fast immer eine restitutio ad integrum erfolgt.

Ist andererseits die Prognose ersichtlich infaust, muß schnellstmöglich eine Transplantation erfolgen. Die Patienten müssen daher frühzeitig in ein Transplantationszentrum verlegt werden, und die Abschätzung der Prognose muß laufend überprüft werden.

\section{Prognosescores}

Bei einigen Erkrankungen ist die Prognose mit Eintritt einer Enzephalopathie Grad 3 oder 4 praktisch immer infaust, so z. B. bei akuter Wilson-Krise oder der Halothanhepatitis. Für andere Erkrankungsgruppen sind Prognosescores entwickelt worden, mit deren Hilfe die Notwendigkeit einer Transplantation frühzeitig erkennbar werden soll (Lake u. Sussman 1995). 
So konnten französische Arbeitsgruppen zeigen, daß ein Absinken des Faktor V auf unter $20 \%$ bei Hepatitis-B-induziertem Leberversagen retrospektiv von keinem der Patienten überlebt worden war. Zusätzliche Berücksichtigung der Konzentration des Alphafetoproteins erhöhte noch die Vorhersagekraft des Scores.

Die ausführlichsten Prognosescores kommen aus England von der Gruppe um Roger Williams. Sie ermöglichen mit Hilfe klinischer und biochemischer Befunde eine Identifizierung von Patienten mit hohem Überlebensrisiko, wobei zwischen „Paracetamolintoxikation“ und „anderen Ursachen" unterschieden wird.

Die positive Vorhersagekraft sowohl der französischen als auch der englischen Scores ist mit über $85 \%$ hoch, jedoch liegt die negative Vorhersagekraft enttäuschend niedrig bei nur etwa $65 \%$, d. h. fast jeder zweite Patient, der laut Prognoseberechnung ohne Transplantation hätte überleben sollen, mußte dennoch transplantiert werden oder verstarb (Anand et al. 1997; s. Übersicht).

\section{Ergebnisse}

Die Ergebnisse der Transplantation sind abhängig vom präoperativen Zustand der Patienten. Prognostisch besonders ungünstig sind Blutungen, Nierenversagen, exzessive Bilirubinerhöhungen und län-

\section{„Kings College Kriterien" für die Indikationsstellung zur Lebertransplantation bei akutem Leberversagen}

Patienten werden mit an Sicherheit grenzender Wahrscheinlichkeit eine Transplantation benötigen, wenn folgende Befunde erhoben werden:

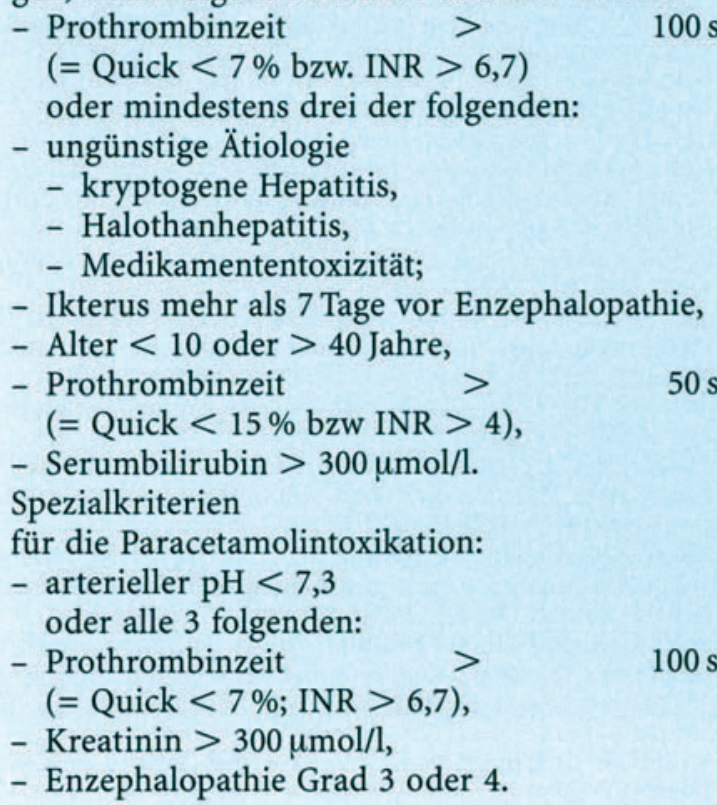

ger bestehende Enzephalopathie Grad 4 (Ascher et al. 1993). Nach Transplantation kann ein vorbestehendes Hirnödem noch bis $\mathrm{zu} 12 \mathrm{~h}$ fortbestehen, und die intrakranielle Druckmessung sollte fortgesetzt werden.

Infektionen. Das Risiko für bakterielle Infektionen und Pilzinfektionen ist nach Transplantation unter der einsetzenden Immunsuppression besonders hoch. Die schlechtere Langzeitüberlebensrate gegenüber anderen Indikationsgruppen bei den tendentiell jüngeren Patienten ist durch die hohe Rate septischer Infektionen mit tödlichem Ausgang bedingt.

\subsection{1 \\ APOLT}

Um Patienten mit potentiell reversiblem Leberversagen die lebenslange Immunsuppression zu ersparen, wurde die Technik der „auxiliären, partiellen orthotopen Lebertransplantation" (APOLT) entwikkelt (Gubernatis et al. 1991). Hierbei wird bei der erkrankten Leber der linke Leberlappen reseziert und durch ein Teiltransplantat ersetzt. Das Transplantat übernimmt die Leberfunktion bis sich die eigene Leber des Empfängers erholt hat. Im Verlauf kann dann die Immunsuppression abgesetzt werden und das Transplantat atrophiert, während die eigene Leber zu normaler Größe hypertrophiert. Solche Teiltransplantationen sind inzwischen weltweit für eine ganze Reihe von Indikationen erfolgreich durchgeführt worden. Das Konzept funktioniert jedoch nur, wenn sich die eigene Leber tatsächlich zügig erholt.

\section{Literatur}

Acharya SK, Dasarathy S, Irshad M (1995) Prospective study of plasma fibronectin in fulminant hepatitis: association with infection and mortality. J Hepatol 23(1): 8-13

Aggarwal S, Kramer D, Yonas H et al. (1994) Cerebral hemodynamic and metabolic changes in fulminant hepatic failure: a retrospective study. Hepatology 19(1): 80-87

Alexander WF, Spindel E, Harty RF et al. (1989) The usefulness of branched chain amino acids in patients with acute or chronic hepatic encephalopathy. Am J Gastroenterol 84(2): 91-96

Anand AC, Nightingale P, Neuberger JM (1997) Early indicators of prognosis in fulminant hepatic failure: an assessment of the King's criteria. J Hepatol 26(1): 62-68

Ascher NL, Lake JR, Emond JC et al. (1993) Liver transplantation for fulminant hepatic failure. Arch Surg 128(6): $677-682$

Baez TA, Gonzalez KC (1994) Acute fatty liver of pregnancy. Case report and review of the literature. P R Health Sci J 13(1): 9-12

Barber JR, Teasley KM (1984) Nutritional support of patients with severe hepatic failure. Clin Pharm 3(3): 245-253 
Belay ED, Bresee JD, Holman RC et al. (1999) Reye's syndrome in the United States from 1981 through 1997. N Engl J Med 340: 1377-1382

Berman DH, Leventhal RI, Gavaler JS et al. (1991) Clinical differentiation of fulminant Wilsonian hepatitis from other causes of hepatic failure. Gastroenterology 100(4): $1129-1134$

Bernuau J, Rueff B, Benhamou JP (1986) Fulminant and subfulminant liver failure: definitions and causes. Semin Liver Dis 6(2): 97-106

Bihari DJ, Gimson AE, Williams R (1986) Cardiovascular, pulmonary and renal complications of fulminant hepatic failure. Semin Liver Dis 6(2): 119-128

Bismuth H, Samuel D, Castaing D et al. (1996) Liver transplantation in Europe for patients with acute liver failure. Semin Liver Dis 16(4): 415-425

Blei AT (1995) Pathogenesis of brain edema in fulminant hepatic failure. Prog Liver Dis 13(311): 311-330

Blei AT, Olafsson S, Webster S et al. (1993) Complications of intracranial pressure monitoring in fulminant hepatic failure . Lancet 341: 157-158

Bray GP, Harrison PM, O'Grady JG et al. (1992) Long-term anticonvulsant therapy worsens outcome in paracetamolinduced fulminant hepatic failure. Hum Exp Toxicol 11(4): 265-270

Cattral MS, Altraif I, Greig PD et al. (1994) Toxic effects of intravenous and oral prostaglandin $\mathrm{E}$ therapy in patients with liver disease. Am J Med 97(4): 369-373

Cordoba J, Blei AT (1995) Cerebral edema and intracranial pressure monitoring. Liver Transpl Surg 1(3): 187-194

Davies MH, Mutimer D, Lowes J et al. (1994) Recovery despite impaired cerebral perfusion in fulminant hepatic failure. Lancet 343: 1329-1330

Davison AM (1996) Hepatorenal failure. Nephrol Dial Transplant 8(24): 24-31

Devlin J, Ellis AE, McPeake J et al. (1997) N-acetylcysteine improves indocyanine green extraction and oxygen transport during hepatic dysfunction. Crit Care Med 25(2): 236-242

Ellis A, Wendon J (1996) Circulatory, respiratory, cerebral, and renal derangements in acute liver failure: pathophysiology and management. Semin Liver Dis 16(4): 379-388

Ellison RT, Perez PG, Welsh CH et al. (1996) Risk factors for upper gastrointestinal bleeding in intensive care unit patients: role of helicobacter pylori. Federal Hyperimmune Immunoglobulin Therapy Study Group. Crit Care Med 24(12): 1974-1981

Feller RB, Wilson JS (1994) Hepatic failure in fatal exertional heatstroke. Aust N Z J Med 24: 69

Fiaccadori F, Pedretti G, Ferrari C et al. (1991) Insulin and glucagon levels in fulminant hepatic failure in man. Dig Dis Sci 36(6): 801-808

Gimson AE (1996) Fulminant and late onset hepatic failure. Br J Anaesth 77(1): 90-98

Gimson A, Tedder R, White Y (1983) Serological markers in fulminant Hepatitis B. Gut 24: 615

Gubernatis G, Pichlmayr R, Kemnitz J et al. (1991) Auxiliary partial orthotopic liver transplantation (APOLT) for fulminant hepatic failure: first successful case report. World J Surg 15(5): 660-665

Gyr K, Meier R (1991) Flumazenil in the treatment of portal systemic encephalopathy- an overview. Intensive Care Med 17 (Suppl 1): S39-S42

Harrison PM, Keays R, Bray GP et al. (1990) Improved outcome of paracetamol-induced fulminant hepatic failure by late administration of acetylcysteine. Lancet 335(8705): $1572-1573$

Häussinger D, Gerok W (1986) The effect of urea synthesis on extracellular $\mathrm{pH}$ in isolated perfused rat liver. Biochem J 236: $261-265$

Hoofnagle JH, Carithers RJ, Shapiro C et al. (1995) Fulminant hepatic failure: summary of a workshop. Hepatology 21(1): $240-252$
Hughes RD, Zhang L, Tsubouchi $\mathrm{H}$ et al. (1994) Plasma hepatocyte growth factor and biliprotein levels and outcome in fulminant hepatic failure. J Hepatol 20(1): 106-111

Irshad M (1997) Hepatitis E virus: a global view of its seroepidemiology and transmission pattern. Trop Gastroenterol 18(2): 45-49

Jain SK, Rohatgi A, Raman KK et al. (1995) Study of serum prealbumin and serum alpha fetoprotein in cases of fulminant hepatic failure. J Assoc Physicians India 43(7): 462-463

Janes J, Routledge PA (1992) Recent developments in the management of paracetamol (acetaminophen) poisoning. Drug Saf 7(3): 170-177

Jauregui HO, Chowdhury NR, Chowdhury JR (1996) Use of mammalian liver cells for artificial liver support. Cell Transplant 5(3): 353-367

Jones EA, Schafer DF, Ferenci P et al. (1984) The GABA hypothesis of the pathogenesis of hepatic encephalopathy: current status. Yale J Biol Med 57(3): 301-316

Jones EA, Basile AS, Skolnick P (1990) Hepatic encephalopathy, GABA-ergic neurotransmission and benzodiazepine receptor ligands. Adv Exp Med Biol 272(121): 121-134

Karvoutzis G, Redecker A, Peters R (1974) Long term follow up of patients surviving fulminant viral hepatitis. Gastroenterology 67: 870

Keays R, Potter D, O’Grady J et al. (1991) Intracranial and cerebral perfusion pressure changes before, during and immediately after orthotopic liver transplantation for fulminant hepatic failure. QJM 79(289): 425-433

Koppel C (1993) Clinical symptomatology and management of mushroom poisoning. Toxicon 31(12): 1513-1540

Krowka MJ (1993) Clinical management of hepatopulmonary syndrome. Sem Liver Dis 13: 414-422

Kuo PC, Johnson LB, Hastings G et al. (1996) Fulminant hepatic failure from the Budd-Chiari syndrome. A bridge to transplantation with transjugular intrahepatic portosystemic shunt. Transplantation 62(2): 294-296

Lake JR, Sussman NL (1995) Determining prognosis in patients with fulminant hepatic failure: when you absolutely, positively have to know the answer. Hepatology 21(3): 879-882

Langley P, Hughes R, Williams R (1985) Increased Faktor VIII complex in fulminant hepaic failure. Thromb Haemost 54: 693-696

Langley PG, Forbes A, Hughes RD et al. (1990) Thrombinantithrombin III complex in fulminant hepatic failure: evidence for disseminated intravascular coagulation and relationship to outcome. Eur J Clin Invest 20(6): 627-631

Langley PG, Keays R, Hughes RD et al. (1991) Antithrombin III supplementation reduces heparin requirement and platelet loss during hemodialysis of patients with fulminant hepatic failure. Hepatology 14(2): 251-256

Larsen FS, Ejlersen E, Clemmesen JO et al. (1996) Preservation of cerebral oxidative metabolism in fulminant hepatic failure: an autoregulation study. Liver Transpl Surg 2(5): 348-353

Lee WM (1996) Management of acute liver failure. Semin Liver Dis 16(4): 369-378

Liang TJ, Hasegawa K, Rimon N et al. (1991) A hepatitis B virus mutant associated with an epidemic of fulminant hepatitis. N Engl J Med 324(24): 1705-1709

Malchesky PS (1994) Nonbiological liver support: historic overview. Artif Organs 18(5): 342-347

McCashland TM, Shaw BJ, Tape E (1996) The American experience with transplantation for acute liver failure. Semin Liver Dis 16(4): 427-433

McGuire BM, Sielaff TD, Nyberg SL et al. (1995) Review of support systems used in the management of fulminant hepatic failure. Dig Dis 13(6): 379-388

Mendez L, Reddy KR, Di PR et al. (1991) Fulminant hepatic failure due to acute hepatitis B and delta co-infection. Am J Gastroenterol 86(7): 895-897

Mendoza A, Fernandez F, Mutimer DJ (1997) Liver transplantation for fulminant hepatic failure: importance of renal failure. Transpl Int 10(1): 55-60 
Meyer RA, Duffy MC (1993) Spontaneous reactivation of chronic hepatitis $\mathrm{B}$ infection leading to fulminant hepatic failure. Report of two cases and review of the literature. J Clin Gastroenterol 17(3): 231-234

Munoz SJ (1993) Difficult management problems in fulminant hepatic failure. Semin Liver Dis 13(4): 395-413

Munoz SJ, Moritz MJ, Martin P et al. (1993) Relationship between cerebral perfusion pressure and systemic hemodynamics in fulminant hepatic failure. Transplant Proc 25(2): 1776-1778

Neuberger JM (1990) Halothane and hepatitis. Incidence, predisposing factors and exposure guidelines. Drug Saf 5(1): 28-38

O'Brien BK (1996) A fatal Sunday brunch: Amanita mushroom poisoning in a Gulf Coast family. Am J Gastroenterol 91(3): 581-583

O'Grady J (1992) Management of acute and fulminant hepatitis A. Vaccine 10 (Suppl 1): S21-S23

O'Grady JG (1996) Pathogenesis of acute liver failure. Trop Gastroenterol 17(4): 199-201

O'Grady JG (1997 a) Paracetamol hepatotoxicity: how to prevent. J R Soc Med 90(7): 368-370

O'Grady JG (1997 b) Paracetamol-induced acute liver failure: prevention and management. J Hepatol 1(41): 41-46

Pandha HS (1992) Treating acidosis in fulminant hepatic failure: the case against use of bicarbonate solutions [letter; comment]. N Z Med J 105: 937

Pereira LM, Langley PG, Hayllar KM et al. (1992) Coagulation factor V and VIII/V ratio as predictors of outcome in paracetamol induced fulminant hepatic failure: relation to other prognostic indicators. Gut 33(1): 98-102

Pereira SP, Langley PG, Williams R (1996) The management of abnormalities of hemostasis in acute liver failure. Semin Liver Dis 16(4): 403-414

Pohl L (1990) Drug induced allergic hepatitis. Semin Liver Dis 10: $305-315$

Riely CA (1994) Hepatic disease in pregnancy. Am J Med 96: $18 \mathrm{~S}-22 \mathrm{~S}$

Rolando N, Wade JJ, Fagan E et al. (1992) An open, comparative trial of aztreonam with vancomycin and gentamicin with piperacillin in patients with fulminant hepatic failure. J Antimicrob Chemother 30(2): 215-220
Rolando N, Philpott HJ, Williams R (1996) Bacterial and fungal infection in acute liver failure. Semin Liver Dis 16(4): 389-402

Sanchez-Tapias J, Mas A, Costa J (1987) Recombinant interferon therapy in fulminant viral hepatitis. J Hepatol 5: 205-210

Stremmel W, Meyerrose KW, Niederau C et al. (1991) Wilson disease: clinical presentation, treatment, and survival. Ann Intern Med 115(9): 720-726

Theilmann L, Solbach C, Toex U et al. (1992) Role of hepatitis $C$ virus infection in German patients with fulminant and subacute hepatic failure. Eur J Clin Invest 22(8): 569-571

Vallbracht A, Gabriel P, Maier K (1986) Cell mediated cytotoxicity in hepatitis A virus infection. Hepatology 6: 1308-1314

Van Pelt F, Straub P, Manns M (1995) Molecular basis of drug induced immunological liver injury. Semin Liver Dis 15: 283-300

Wendon JA, Harrison PM, Keays R et al. (1992) Effects of vasopressor agents and epoprostenol on systemic hemodynamics and oxygen transport in fulminant hepatic failure. Hepatology 15(6): 1067-1071

Wijdicks EF, Plevak DJ, Rakela J et al. (1995) Clinical and radiologic features of cerebral edema in fulminant hepatic failure. Mayo Clin Proc 70(2): 119-124

Williams R (1996) Classification, etiology, and considerations of outcome in acute liver failure. Semin Liver Dis 16(4): 343-348

Williams R, Gimson AE (1991) Intensive liver care and management of acute hepatic failure. Dig Dis Sci 36(6): $820-826$

Williams R, Wendon J (1994) Indications for orthotopic liver transplantation in fulminant liver failure. Hepatology 20: $5 S-10 S$

Williams R, O'Grady JG, Davies SE et al. (1992) Liver transplantation and hepatitis viruses. Arch Virol Suppl 4(11): 311-316

Woolf GM, Redeker AG (1991) Treatment of fulminant hepatic failure with insulin and glucagon. A randomized, controlled trial. Dig Dis Sci 36(1): 92-96

Wyke RJ (1989) Bacterial infections complicating liver disease. Baillieres Clin Gastroenterol 3(1): 187-210 